\title{
HLA-DQ Antigen
}

National Cancer Institute

\section{Source}

National Cancer Institute. HLA-DQ Antigen. NCI Thesaurus. Code C62792.

HLA-DQ antigen is a class of glycoproteins that consist of dimers of alpha and beta subunits, which are encoded by the polymorphic human genes HLA-DQA1 and HLADQB1, respectively. This protein complex is expressed primarily in lymphoid cells and function to present antigens for CD4+ T lymphocytes. Certain HLA-DQ protein variants are associated with susceptibility to insulin-dependent diabetes mellitus and several autoimmune diseases. 